\title{
Eupafolin alleviates cerebral ischemia/reperfusion injury in rats via blocking the TLR4/NF-кB signaling pathway
}

\author{
XINGWANG CHEN, ZHIJUN YAO, XIAN PENG, LONG WU, HUACHU WU, YUANTONG OU and JIANBO LAI \\ Department of Intensive Care Unit, Shenzhen Hospital of Integrated Traditional Chinese and Western Medicine, \\ Shenzhen, Guangdong 518104, P.R. China
}

Received June 18, 2020; Accepted September 11, 2020

DOI: $10.3892 / \mathrm{mmr} .2020 .11637$

\begin{abstract}
Eupatorium perfoliatum L. (E. perfoliatium) has been used traditionally for treating fever, malaria and inflammation-associated diseases. Eupafolin, the extract of E. perfoliatium, was also reported to suppress inflammation. The present study aimed to investigate the protective effects of eupafolin on cerebral ischemia/reperfusion (I/R) injury in rats and its possible underlying mechanisms. Cerebral I/R injury was induced in rats by middle cerebral artery occlusion (MCAO) for $1.5 \mathrm{~h}$, followed by reperfusion. The rats were randomly assigned into six groups: Control, model, $10 \mathrm{mg} / \mathrm{kg}$ eupafolin, $20 \mathrm{mg} / \mathrm{kg}$ eupafolin, $50 \mathrm{mg} / \mathrm{kg}$ eupafolin and $20 \mathrm{mg} / \mathrm{kg}$ nimodipine. Eupafolin and nimodipine were intragastrically administrated to the rats 1 week before MCAO induction. Following reperfusion for $24 \mathrm{~h}$, the neurological deficit was scored, and brain samples were harvested for evaluating encephaledema, infarct volume, oxidative stress, apoptosis, inflammation and the expression of TLR4/NF- $\kappa \mathrm{B}$ signaling. The results revealed that eupafolin decreased the neurological score, relieved encephaledema and decreased infarct volume. Eupafolin also attenuated oxidative stress, neuronal apoptosis and inflammation, with decreases in lactate dehydrogenase, malondialdehyde, TUNEL-positive cells, Bax and caspase-3, along with TNF- $\alpha$, IL-1 $\beta$ and IL-6, but increases in superoxide dismutase and Bcl-2 levels. Furthermore, eupafolin may decrease the expression of TLR4 downstream proteins and proteins involved in the $\mathrm{NF}-\kappa \mathrm{B}$ pathway. Treatment with TLR4 agonist-LPS significantly blunted the protective effect of eupafolin on encephaledema and cerebral infarct. Meanwhile, $20 \mathrm{mg} / \mathrm{kg}$ eupafolin showed nearly equivalent effects to the positive-control drug nimodipine. In conclusion, eupafolin protected against cerebral I/R injury in rats and the
\end{abstract}

Correspondence to: Dr Jianbo Lai, Department of Intensive Care Unit, Shenzhen Hospital of Integrated Traditional Chinese and Western Medicine, 3 Shajing Street, Shenzhen, Guangdong 518104, P.R. China

E-mail: laijianbo20@163.com

Key words: cerebral ischemia reperfusion, eupafolin, inflammation, nuclear factor- $\kappa \mathrm{B}$, toll-like receptor underlying mechanism may be associated with the suppression of apoptosis and inflammation via inhibiting the TLR4/ $\mathrm{NF}-\kappa \mathrm{B}$ signaling pathway.

\section{Introduction}

Ischemic stroke remains a leading cause of mortality or long-term disability. The goal of clinical treatment is to restore the blood supply as soon as possible, allowing timely supply of oxygen to ischemic brain tissue (1). However, blood reperfusion that occurs after a long period of ischemia is likely to result in a higher infarction volume and to, in turn, aggravate the initial injury, which is the main cause of cerebral ischemia reperfusion (I/R) injury (2). The mechanisms involved in cerebral I/R is complicated and require comprehensive understanding. To date, inflammatory response, free radical damage, cytotoxicity, increased mitochondrial permeability and intracellular calcium overload have been implicated to participate in the occurrence or progression of cerebral I/R injury (3). Cerebral I/R injury is pathologically characterized by the damage of brain tissues, infiltration of leukocyte cells into the brain, influx of inflammatory cells, excessive production of reactive oxygen species (ROS), degradation of cytoskeletal protein and collapse of the blood-brain barrier (4). Increased ROS production following reperfusion will increase hemorrhagic infarction, cerebral edema and infarct size (5). Inhibition of oxidative stress has been demonstrated to protect against cerebral I/R injury $(6,7)$. Therefore, the use of safe and effective therapeutic agents with antioxidant properties to interfere with oxidative damage provides an encouraging treatment strategy.

Eupafolin is an active flavonoid component of Eupatorium perfoliatum $L$., which is a traditional herbal medicine from China and India, and has been widely used for centuries to treat fever, malaria, infections and inflammation-associated diseases (8). In recent years, eupafolin was reported to exhibit anti-inflammatory, anti-oxidant and anti-tumor cell proliferation effects (9). For example, Zhang et al (10) indicated that eupafolin improved acute renal injury and exhibited effective anti-oxidant and anti-inflammatory activities via inhibiting reactive stress and inactivating NF- $\mathrm{B}$, MAPK, ERK 1/2 and c-JNK signaling pathways (10). Eupafolin ameliorated cardiomyocyte autophagy via activation of the PI3K/AKT/mTOR signaling pathway (11). Eupafolin also protected against TNF- $\alpha$-induced lung inflammation via inhibiting NF- $\mathrm{N} / \mathrm{p} 65$ 
activation and also resulted in nuclear translocation (12). In addition, the suppressive effects of eupafolin on various tumor types, including esophageal cancer, hepatocellular carcinoma, renal carcinoma and prostate cancer has been extensively studied (13-16). These data suggested the potential value of eupafolin in treating cancer and inflammation-related diseases. However, whether eupafolin may protect the brain against I/R injury remains to be elucidated.

Toll-like receptor (TLR) is a type of transmembrane protein that converts extracellular antigen information into cells and triggers an inflammatory response. Toll-like receptor 4 (TLR4) is the first discovered member of TLR, which serves a role in immune defense and immune regulation by recognizing and binding multiple endogenous and exogenous ligands. TLR4 transduces these signals via the membrane and subsequently regulates the expression inflammation mediators and cytokines (17). A previous study demonstrated that TLR4 serves a role in I/R inflammation injury of the liver, lung and heart, and induces apoptosis during cerebral I/R (18). NF- $\mathrm{BB}$ is a transcription factor that may specifically bind promoters and enhancers of numerous genes and thus participate in a variety of cellular functions, including inflammation, cell proliferation and apoptosis. NF- $\kappa \mathrm{B}$ is typically inactivated in the cytoplasm due to the interaction of p65/p52 with the inhibitory protein $\mathrm{I} \kappa \mathrm{B}$. The subsequent activation of $\mathrm{p} 65 / \mathrm{p} 52$ may be stimulated by pro-inflammatory cytokines, cellular stress, DNA damaging agents and phosphorylation of $\kappa$ Bs by the I $\mathrm{B}$ kinase complex (19). NF- $\mathrm{B}$ also serves a crucial role in the activation of $\mathrm{I} / \mathrm{R}$ injury (20).

The present study investigated the protective effects of eupafolin against cerebral I/R injury in rats and investigated whether its action was dependent on blocking the TLR4/NF- $\kappa B$ signaling pathway.

\section{Materials and methods}

Animals. Adult male Sprague-Dawley rats (7-8 week-old; $\mathrm{n}=48$ ) weighing 250-280 g were supplied by Nanjing Better Biotechnology Co., Ltd, and were acclimatized for 1 week before experiments at room temperature under a controlled 12/12 h light/dark cycle. All rats received food and water ad libitum. The experimental protocols involving rats were approved by the Animal Studies Ethics Committee of the Shenzhen Hospital of Integrated Traditional Chinese and Western Medicine.

The animals were randomly assigned to six groups $(n=8$ for each group): Control, model, $10 \mathrm{mg} / \mathrm{kg}$ eupafolin (purity $>98 \%$; Shanghai Rongbia Biological Technology Co., Ltd.), $20 \mathrm{mg} / \mathrm{kg}$ eupafolin, $50 \mathrm{mg} / \mathrm{kg}$ eupafolin and $20 \mathrm{mg} / \mathrm{kg}$ nimodipine (MedChemExpress). The control group underwent sham surgery. Eupafolin and nimodipine were intragastrically (i.g.) administered into the rats once a day for 7 consecutive days. The control and model groups were administered $200 \mu \mathrm{l}$ normal saline i.g. For the eupafolin + lipopolysaccharide (LPS) group, the animals were intraperitoneally injected with $50 \mu \mathrm{g} / \mathrm{kg}$ LPS (21) at the same time that they received eupafolin $20 \mathrm{mg} / \mathrm{kg}$ for the last time.

Establishment of a cerebral I/R model. One hour after the last administration, the focal cerebral I/R rat model was prepared using the middle cerebral artery occlusion (MCAO) method, as previously reported with slight modifications (22). In brief, following weighing, the rats were anesthetized with $1 \%$ pentobarbital (50 mg/kg; i.p) and fixed in the supine position on a heated operating table with the body temperature maintained at $37 \pm 0.5^{\circ} \mathrm{C}$. Following skin incision, the left common carotid artery, external carotid artery and internal carotid artery were carefully exposed and dissected away from the adjacent nerve. The left middle cerebral artery was occluded by inserting a 3.5-mm monofilament suture through the internal carotid artery from the external carotid artery. Following ischemia for $1.5 \mathrm{~h}$, the suture was gently withdrawn allowing reperfusion. At $24 \mathrm{~h}$ post-reperfusion, various indexes were measured (23). The animals in the sham-operated group were anesthetized with $1 \%$ pentobarbital $(50 \mathrm{mg} / \mathrm{kg}$; i.p) prior to being subjected to the same surgical procedure as the model group but without occlusion of the middle cerebral artery (24).

Neurological score. The neurological deficit was scored in each mouse $24 \mathrm{~h}$ after reperfusion in a blinded manner by two independent investigators according to the 3-point scoring system of Bederson et al (25): No neurological symptoms=0; forelimb flexion and no other abnormality $=1$; decreased resistance to lateral push (and forelimb flexion) without circling=2; same behavior as grade 2, with circling toward the paretic side $=3$.

Measurement of brain edema. To evaluate brain edema, the brain water content was measured using the standard wet-dry method. After $24 \mathrm{~h}$ of reperfusion, the rats were decapitated under deep anesthesia and the brains were carefully removed. The wet weight was obtained immediately by weighing the ischemic hemispheres, and the tissues were dried at $100^{\circ} \mathrm{C}$ for $24 \mathrm{~h}$ to determine the dry weight. The degree of brain edema was calculated using the following equation: Water content $=$ (wet weight-dry weight $) /$ wet weight $\mathrm{x} 100 \%$; brain index=wet weight/body weight $\mathrm{x} 100 \%$.

Measurement of infract volume. Brain infarction size was evaluated by the 2,3,5-triphenyltetrazolium chloride (TTC) staining method (26) $24 \mathrm{~h}$ after I/R. Brains were carefully removed and maintained at $-20^{\circ} \mathrm{C}$ for $10 \mathrm{~min}$. Brain tissues were then sliced into consecutive 2 -mm-thick coronal sections and immersed in 2\% TTC solution (Sigma-Aldrich; Merck $\mathrm{KGaA}$ ) for $30 \mathrm{~min}$ at $37^{\circ} \mathrm{C}$. TTC stains non-infarcted regions with a deep red pigment, while the infarcted brain area appears white. The infarct area of each section was photographed and image analysis software (NIH Image version 1.63; National Institutes of Health) was applied to measure the infarcted area.

Measurement of oxidative stress. The fresh brain tissues of rats at $24 \mathrm{~h}$ after cerebral $\mathrm{I} / \mathrm{R}$ were collected and the superoxide dismutase (SOD; cat. no. ab65354), malondialdehyde levels (MDA; cat. no. ab118970) and lactate dehydrogenase (LDH; cat. no. ab102526) activities in the brain tissues were determined using the commercial kits (Abcam). The brain tissues were harvested, washed with PBS and homogenized using RIPA lysis buffer supplemented with PMSF protease inhibitor (both from Abcam). After being centrifuged at 13,000 x g and $4^{\circ} \mathrm{C}$ for $10 \mathrm{~min}$ to remove insoluble material, the supernatants were collected and incubated with corresponding reaction 
mix according to the manufacturer's protocols. The optical density was measured (OD of $450 \mathrm{~nm}$ for SOD and LDH; OD of $532 \mathrm{~nm}$ for MDA) to calculate the relative level of SOD, MDA and LDH. The relative MDA levels, as well as SOD and LDH activities was expressed as the value dividing by the OD of the control group after normalization to the standard curve.

Enzyme-linked immunosorbent assay (ELISA). The concentrations of TNF- $\alpha$, IL- $1 \beta$ and IL- 6 in the serum of rats at $24 \mathrm{~h}$ after cerebral $\mathrm{I} / \mathrm{R}$ were measured in strict accordance with the manufacturer's protocols provided by the ELISA kits (Abcam) for TNF- $\alpha$ (cat. no. ab236712), IL-1 $\beta$ (cat. no. ab255730) and IL-6 (cat. no. ab234570). In brief, blood samples were collected into a serum separator tube. Following clot formation, samples were centrifuged at $2,000 \mathrm{x} \mathrm{g}$ and $4^{\circ} \mathrm{C}$ for $10 \mathrm{~min}$ to collect serum. Samples were incubated with antibodies (included in the kits) targeting TNF- $\alpha$, IL- $1 \beta$ and IL- 6 at room temperature for $1 \mathrm{~h}$. Following washing with the supplied wash buffer, TMB development solution was added and incubated at room temperature for $10 \mathrm{~min}$. The stop solution was added and the absorbance at a wavelength of $450 \mathrm{~nm}$ was detected using a microplate reader (Thermo Fisher Scientific, Inc.).

TUNEL staining. A TUNEL assay (Beyotime Institute of Biotechnology) was used according to the manufacturer's protocols to assess neuronal apoptosis in brain tissues. In brief, isolated brains were fixed in $4 \%$ paraformaldehyde at room temperature for $10 \mathrm{~min}$ and cut into sections of $20-\mu \mathrm{m}$ thickness, followed by paraffin embedding. Following dewaxing with xylene, sections were incubated with protease $\mathrm{K}$ for $30 \mathrm{~min}$ and subsequently washed with phosphate-buffered saline (PBS). Subsequently, $50 \mu 1$ TUNEL reaction mixture was added and incubated for $1 \mathrm{~h}$ at $37^{\circ} \mathrm{C}$. The sections were then washed with PBS and incubated for $30 \mathrm{~min}$ following the addition of $50 \mu \mathrm{l}$ confining liquid. The nuclei were stained with DAPI at room temperature for $5 \mathrm{~min}$ and TUNEL-positive cells were observed under a DMi8 fluorescence microscope (Leica Microsystems $\mathrm{GmbH}$ ). Three fields of view were examined (magnification, $\mathrm{x} 200$ ).

Western blotting. The ischemic side of the cerebral cortex was dissected to extract total protein using RIPA buffer (Applygen Technologies Inc.) and quantified using a BCA assay. Following quantification, equal amount of proteins $(8 \mu \mathrm{g})$ were separated by $10 \%$ SDS-PAGE and transferred onto polyvinylidene difluoride membranes (EMD Millipore). Following blocking with 5\% skimmed milk at room temperature for $2 \mathrm{~h}$, the membranes were incubated with the following primary antibodies at $4^{\circ} \mathrm{C}$ overnight (all Abcam): Bcl-2 (cat. no. ab32124; dilution, 1:1,000), Bax (cat. no. ab32503; dilution, 1:5,000), pro-caspase 3 (cat. no. ab32499; dilution, 1:10,000), cleaved-caspase 3 (cat. no. ab32024; dilution, 1:500), myeloid differentiation factor 88 (MyD88; cat. no. ab133739; dilution, 1:10,000), TNF receptor-associated factor 6 (TRAF6; cat. no. ab33915; dilution, 1:10,000), TGF- $\beta$-activated kinase 1 (TAK1; cat. no. ab109526; dilution, 1:1,000), IKK $\alpha$ (cat. no. ab32041; dilution, 1:10,000), p65 (cat. no. ab16502; dilution, 1:5,000), phosphorylated (p)-IKK $\alpha$ (cat. no. ab38515; dilution, 1:1,000), p-p65 (cat.no. ab183559; dilution, 1:1,000) and GAPDH (cat. no. ab8245; dilution, 1: 10,000). The antibodies
Table I. Effect of eupafolin on the neurological deficit score.

Group Neurological deficit scores (range, 0-3)

\section{Control}

$0.00 \pm 0.00$

Model

$2.63 \pm 0.51^{\mathrm{a}}$

Eupafolin $(10 \mathrm{mg} / \mathrm{kg})$

$2.13 \pm 0.31^{\mathrm{b}}$

Eupafolin $(20 \mathrm{mg} / \mathrm{kg})$

$1.10 \pm 0.43^{\mathrm{c}}$

Eupafolin $(50 \mathrm{mg} / \mathrm{kg})$

$0.85 \pm 0.17^{\mathrm{c}}$

Nimodipine

$1.13 \pm 0.24^{\mathrm{c}}$

The neurological deficit scores of rats among the Control, Model, $10 \mathrm{mg} / \mathrm{kg}$ eupafolin, $20 \mathrm{mg} / \mathrm{kg}$ eupafolin, $50 \mathrm{mg} / \mathrm{kg}$ eupafolin and nimodipine groups were measured at $24 \mathrm{~h}$ after cerebral $\mathrm{I} / \mathrm{R}(\mathrm{n}=8)$. Data are presented the mean \pm standard deviation. ${ }^{\mathrm{a}} \mathrm{P}<0.001$ vs. Control group; ${ }^{\mathrm{b}} \mathrm{P}<0.05$ and ${ }^{\mathrm{c}} \mathrm{P}<0.001$ vs. Model group.

were detected using horseradish peroxidase-conjugated IgG (Abcam; goat anti-rabbit, cat. no. ab7090; dilution, 1:10,000) at room temperature for $2 \mathrm{~h}$ and visualized using enhanced chemiluminescence (Thermo Fisher Scientific, Inc.).

Statistical analysis. Data are expressed as the mean \pm standard deviation. SPSS (version 22.0, IBM Corp.) was used to perform the paired $\mathrm{T}$ test between two groups and one-way analysis of variance, followed by Tukey's test. $\mathrm{P}<0.05$ was considered to indicate a statistically significant difference.

\section{Results}

Effects of eupafolin on the neurological defect score, brain edema and infract volume in rats subjected to cerebral I/R. The alteration in neurological functions and phenotypes was first investigated. The effects of eupafolin on the neurological defect scores at $24 \mathrm{~h}$ post-reperfusion in the control, model, eupafolin $(10,20$ and $50 \mathrm{mg} / \mathrm{kg})$ and nimodipine $(20 \mathrm{mg} / \mathrm{kg})$ groups are presented in Table I. Rats in the control group did not exhibit neurological deficits, with scores of 0 . However, in the model group, there were clear signs of neurological deficits, including forelimb flexion, decreased resistance to lateral push and circling toward the paretic side, with significantly higher neurological defect scores compared with the control group, indicating the occurrence of cerebral I/R injury. Eupafolin at any dose significantly decreased the neurological defect scores compared with the model group, similar to that in the nimodipine group.

The results of the water content and brain index studies were consistent with the trend observed for the neurological defect scores. As shown in Table II, the brain water content and brain index of the rats were increased in the model group, while those in the low, moderate and high-dose eupafolin and nimodipine groups were significantly lower those in the model group.

The cerebral infarct volumes were assessed using TTC staining. As shown in Fig. 1, no infarction (white staining) was found in the control group. Compared with the control group, cerebral I/R injury induced significant infarction in the model group. However, treatment with eupafolin at 10 , 
Table II. Effect of eupafolin on brain edema.

\begin{tabular}{lcc}
\hline Group & Brain water content, $\%$ & Brain index, \% \\
\hline Control & $82.58 \pm 0.44$ & $0.52 \pm 0.02$ \\
Model & $84.10 \pm 0.51^{\mathrm{a}}$ & $0.72 \pm 0.04^{\mathrm{a}}$ \\
Eupafolin $(10 \mathrm{mg} / \mathrm{kg})$ & $83.41 \pm 0.41^{\mathrm{b}}$ & $0.68 \pm 0.02^{\mathrm{b}}$ \\
Eupafolin $(20 \mathrm{mg} / \mathrm{kg})$ & $82.90 \pm 0.43^{\mathrm{c}}$ & $0.60 \pm 0.03^{\mathrm{d}}$ \\
Eupafolin $(50 \mathrm{mg} / \mathrm{kg})$ & $82.60 \pm 0.38^{\mathrm{c}}$ & $0.57 \pm 0.01^{\mathrm{d}}$ \\
Nimodipine & $82.94 \pm 0.34^{\mathrm{c}}$ & $0.63 \pm 0.03^{\mathrm{d}}$
\end{tabular}

The brain samples of each group were harvested at $24 \mathrm{~h}$ after cerebral $\mathrm{I} / \mathrm{R}$, and then the brain water content and brain index were measured $(\mathrm{n}=8)$. Data are presented as the mean \pm standard deviation. ${ }^{\mathrm{a}} \mathrm{P}<0.001 \mathrm{vs}$. Control group; ${ }^{\mathrm{b}} \mathrm{P}<0.05,{ }^{\mathrm{c}} \mathrm{P}<0.01$ and ${ }^{\mathrm{d}} \mathrm{P}<0.001$ vs. Model group.

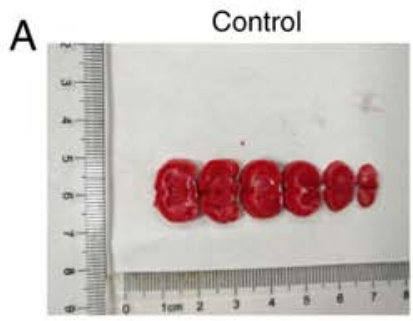

Eupafolin $(20 \mathrm{mg} / \mathrm{kg})$

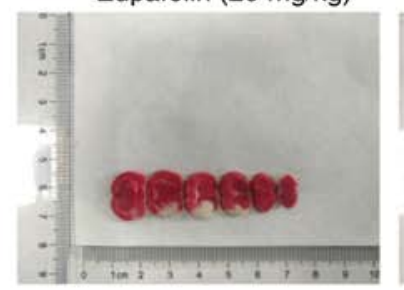

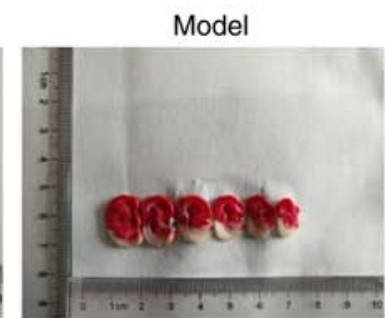

Eupafolin $(50 \mathrm{mg} / \mathrm{kg})$

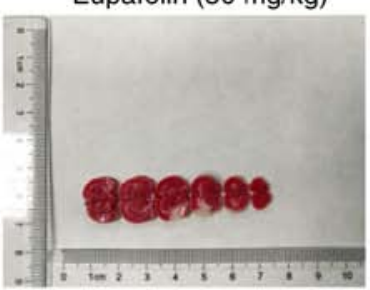

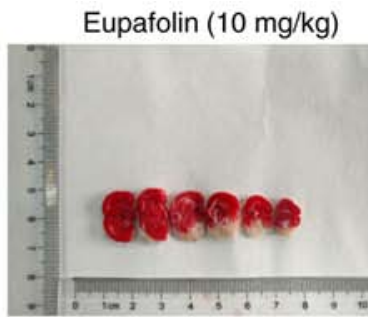

Nemodipine

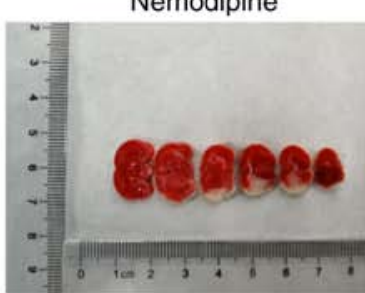

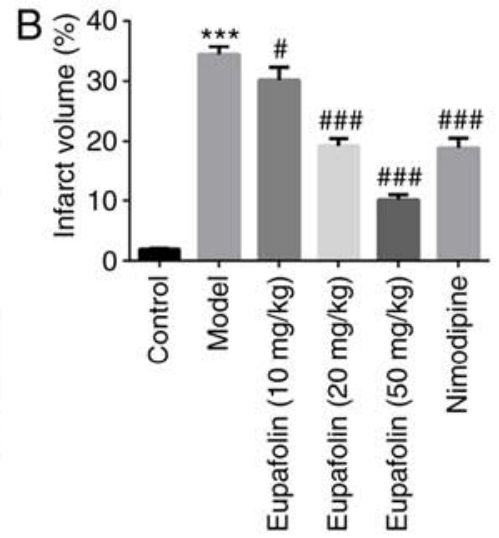

Figure 1. Cerebral infarction volume of rats from control, model, $10 \mathrm{mg} / \mathrm{kg}$ eupafolin, $20 \mathrm{mg} / \mathrm{kg}$ eupafolin, $50 \mathrm{mg} / \mathrm{kg}$ eupafolin and nimodipine groups. (A) TCC staining images of brain tissues in rats at $24 \mathrm{~h}$ after cerebral I/R. (B) Histogram of cerebral infarction volume in different groups. ${ }^{* * *} \mathrm{P}<0.001 \mathrm{vs}$. control. ${ }^{\#} \mathrm{P}<0.05$ and ${ }^{\# \# \#} \mathrm{P}<0.001$ vs. model. I/R, ischemia/reperfusion; TTC, 2,3,5-triphenyltetrazolium chloride.

20 and $50 \mathrm{mg} / \mathrm{kg}$ markedly decreased the infarct volume of brain tissues. Nimodipine exerted similar effects in terms of decreasing the infarct volumes.

Eupafolin decreases oxidative stress and inflammation in brain tissues and serum of rats subjected to cerebral I/R. Subsequently, oxidative stress was examined in ischemic brain tissues, which is considered the initial step of cerebral I/R injury. SOD is an important antioxidant enzyme, while MDA and LDH activities reflect oxidative damage. Therefore, SOD, MDA and LDH contents were investigated (Fig. 2A-C). Compared with the control group, SOD activity was significantly decreased, while MDA and LDH content was increased in the model group. Treatment with eupafolin effectively increased SOD activity and decreased MDA and LDH content, which was similar to the results in the nimodipine group.

To investigate the effects of eupafolin on inflammation, the generation of pro-inflammatory cytokines including TNF- $\alpha$, IL-1 $\beta$ and IL-6 were assessed (Fig. 2D-F). Cerebral I/R injury significantly increased the concentration of TNF- $\alpha$, IL- $1 \beta$ and IL- 6 in the serum of model rats. However, this increase was reversed by eupafolin or nimodipine treatment. The aforementioned findings suggested that, similar to nimodipine, eupafolin attenuated oxidative stress and inflammation induced by cerebral I/R injury.

Eupafolin prevents brain cell apoptosis in rats subjected to cerebral $I / R$. Next, the effects of eupafolin on cell apoptosis were observed by TUNEL staining and western blotting. As shown in Fig. 3, the number of TUNEL-positive cells in the brain tissues of the model group was significantly increased, compared with the control group. These effects were significantly impaired by eupafolin or nimodipine treatment. Furthermore, western blotting was utilized to detect the expression of proteins associated with apoptosis. The results revealed that cerebral I/R lead to an enhancement in Bax and caspase- 3 expression, but a decline in the anti-apoptotic protein Bcl-2 expression (Fig. 4). However, eupafolin and nimodipine partially reversed the levels of these proteins, compared with the model group. Consistent with TUNEL staining, these results demonstrated that eupafolin may prevent cerebral I/R-induced cell apoptosis in the brain tissues of rats. 

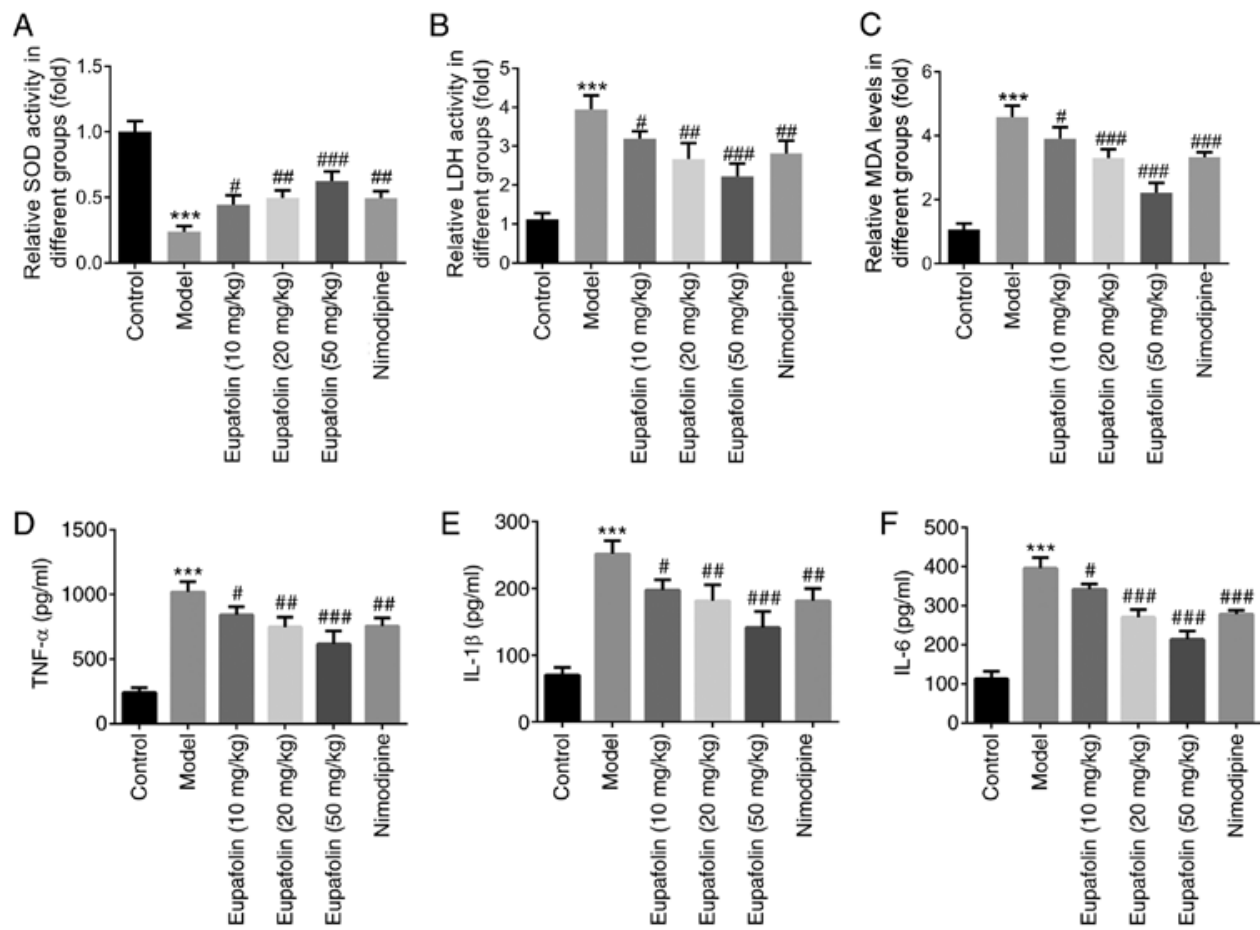

Figure 2. Levels of oxidative stress and inflammation in rats from control, model, $10 \mathrm{mg} / \mathrm{kg}$ eupafolin, $20 \mathrm{mg} / \mathrm{kg}$ eupafolin, $50 \mathrm{mg} / \mathrm{kg}$ eupafolin and nimodipine groups. The activities of (A) SOD, (B) MDA and (C) LDH in the brain tissues of rats at $24 \mathrm{~h}$ after cerebral I/R. The concentrations of (D) TNF- $\alpha$, (E) IL-1 $\beta$ and (F) IL-6 in the serum of rats at $24 \mathrm{~h}$ after cerebral I/R. ${ }^{* * *} \mathrm{P}<0.001 \mathrm{vs}$. control. ${ }^{\#} \mathrm{P}<0.05,{ }^{\# \#} \mathrm{P}<0.01$ and ${ }^{\# \#} \mathrm{P}<0.001$ vs. model. SOD, superoxide dismutase; MDA, malondialdehyde; LDH, lactate dehydrogenase; I/R, ischemia/reperfusion.

A
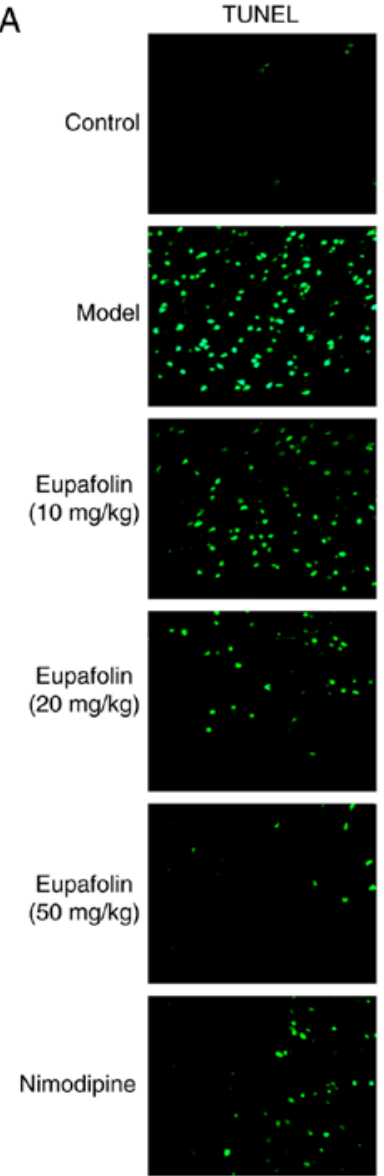

DAPI
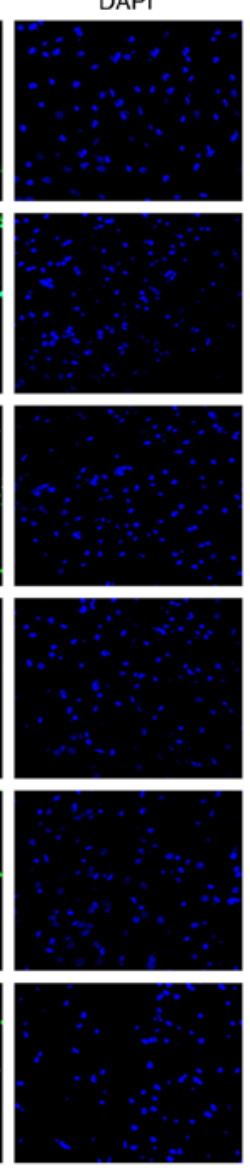

Merge
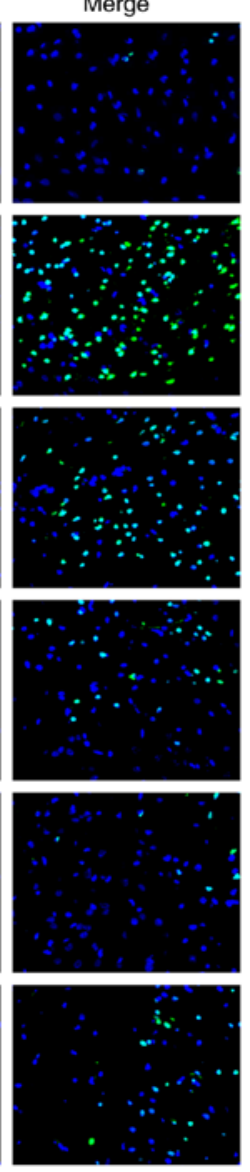

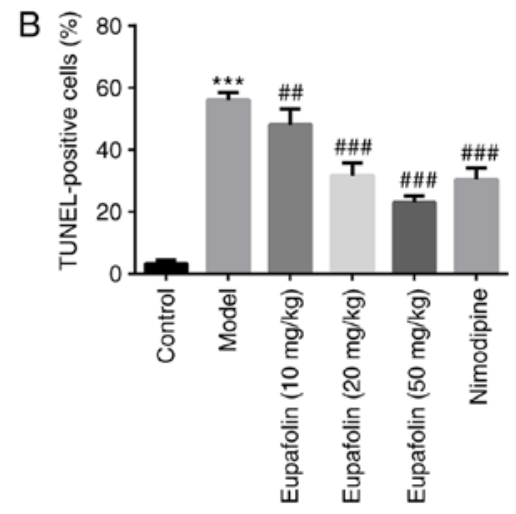

Figure 3. Percentage of apoptotic cells in rats from control, model, $10 \mathrm{mg} / \mathrm{kg}$ eupafolin, $20 \mathrm{mg} / \mathrm{kg}$ eupafolin, $50 \mathrm{mg} / \mathrm{kg}$ eupafolin and nimodipine groups (A) Representative TUNEL staining images to determine cell apoptosis in brain tissues of rats at $24 \mathrm{~h}$ after cerebral I/R. (B) Percentage of TUNEL-positive cells in different groups. ${ }^{* * *} \mathrm{P}<0.001$ vs. control. ${ }^{\# \#} \mathrm{P}<0.05$ and ${ }^{\# \# \#} \mathrm{P}<0.001$ vs. model. I/R, ischemia/reperfusion. 
A
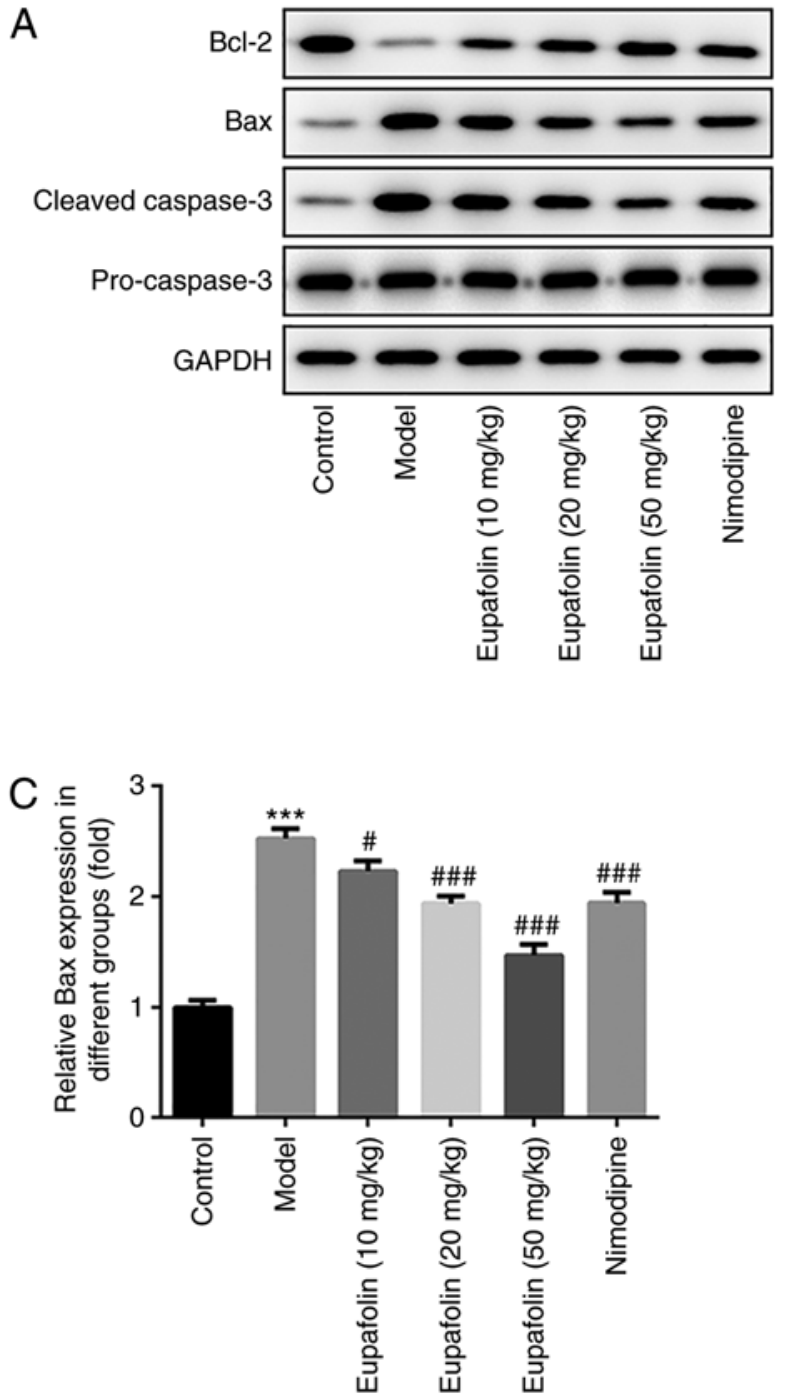
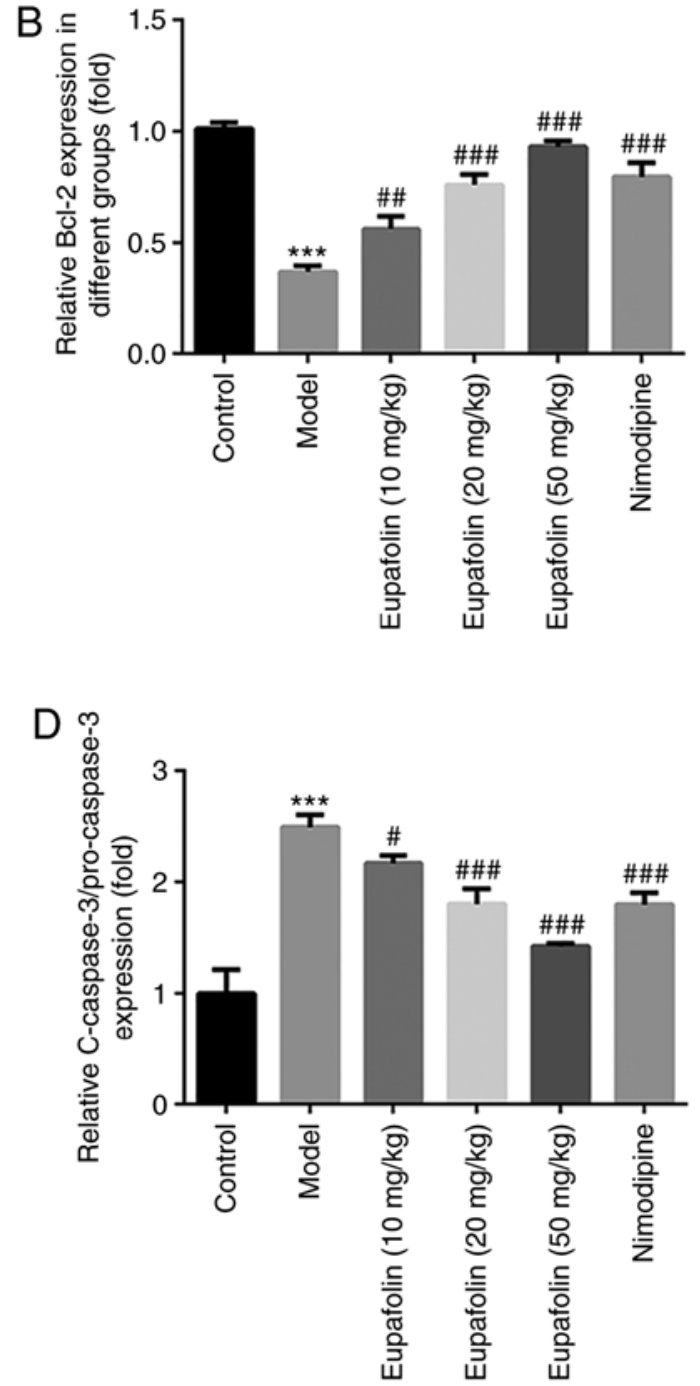

Figure 4. Expression of proteins involved in cell apoptosis in brain tissues of rats from control, $\mathrm{model}, 10 \mathrm{mg} / \mathrm{kg}$ eupafolin, $20 \mathrm{mg} / \mathrm{kg}$ eupafolin, $50 \mathrm{mg} / \mathrm{kg}$ eupafolin and nimodipine groups. (A) Representative western blot bands for detecting Bcl-2, Bax, pro-caspase 3 and cleaved-caspase 3. GAPDH was used as the loading control. Densitometric quantification of (B) Bcl-2, (C) Bax and (D) cleaved-caspase 3/pro-caspase 3 expression. ${ }^{* * *} \mathrm{P}<0.001$ vs. control. ${ }^{*} \mathrm{P}<0.05$, ${ }^{\# \#} \mathrm{P}<0.01$ and ${ }^{\# \# \#} \mathrm{P}<0.001$ vs. model.

Eupafolin inhibits the activation of the TLR4/NF- $\kappa$ B signaling pathway. Finally, to underline the possible mechanism of eupafolin protection against cerebral I/R injury, the expression of proteins involved in TLR4/NF- $\mathrm{KB}$ signaling, including MyD88, TRAF6, TAK1, IKK $\alpha$ and p65, in brain tissues were examined. As shown in Fig. 5, in the model group, the expression of MyD88, TRAF6, TAK1, p-IKK $\alpha$ and p65 was significantly higher than that in the control group, suggesting the activation of TLR4 signaling and nuclear translocation of the NF- $\kappa B$ complex. However, eupafolin $(10,20$ and $50 \mathrm{mg} / \mathrm{kg})$ or nimodipine $(20 \mathrm{mg} / \mathrm{kg})$ significantly inhibited the expression of these proteins.

To verify whether the protective effects of eupafolin rely on blocking TLR4 signaling, animals that were treated with $20 \mathrm{mg} / \mathrm{kg}$ eupafolin were injected with LPS, which is the agonist of TLR4 (21). Table III and Fig. 6 demonstrate that, compared with the eupafolin groups, following MCAO and reperfusion, animals in the eupafolin + LPS group exhibited markedly higher neurological deficit scores, brain water content and brain indexes (Table III), as well as larger infract volume (Fig. 6).

\section{Discussion}

In the present study, treatment of rats subjected to cerebral $\mathrm{I} / \mathrm{R}$ with eupafolin not only decreased the neurological deficit score, brain edema and cerebral infarct size, but also weakened oxidative stress, inflammation and cell apoptosis. This protection by eupafolin against cerebral I/R injury was accompanied by the downregulation of MyD88, TRAF6, TAK1, $\mathrm{p}-\mathrm{IKK} \alpha$ and $\mathrm{p}-\mathrm{p} 65$. These results indicated that TLR4/NF- $\mathrm{KB}$ pathways are inactivated in this process, implying that the TLR4/NF- $\kappa \mathrm{B}$ pathways are involved in the neuroprotective effects of eupafolin.

Previous studies have shown that inflammation, oxidative stress and apoptosis are the three dominating mechanisms underlying the detrimental process of cerebral I/R injury, which was proven to be the second most common lethal factor and the leading cause of adult neurological disabilities worldwide $(3,4,27,28)$. In addition, inflammation is one of the central preventive mechanisms against cerebral I/R injury $(3,4)$. Therefore, inhibiting neuronal inflammation is a pivotal target 

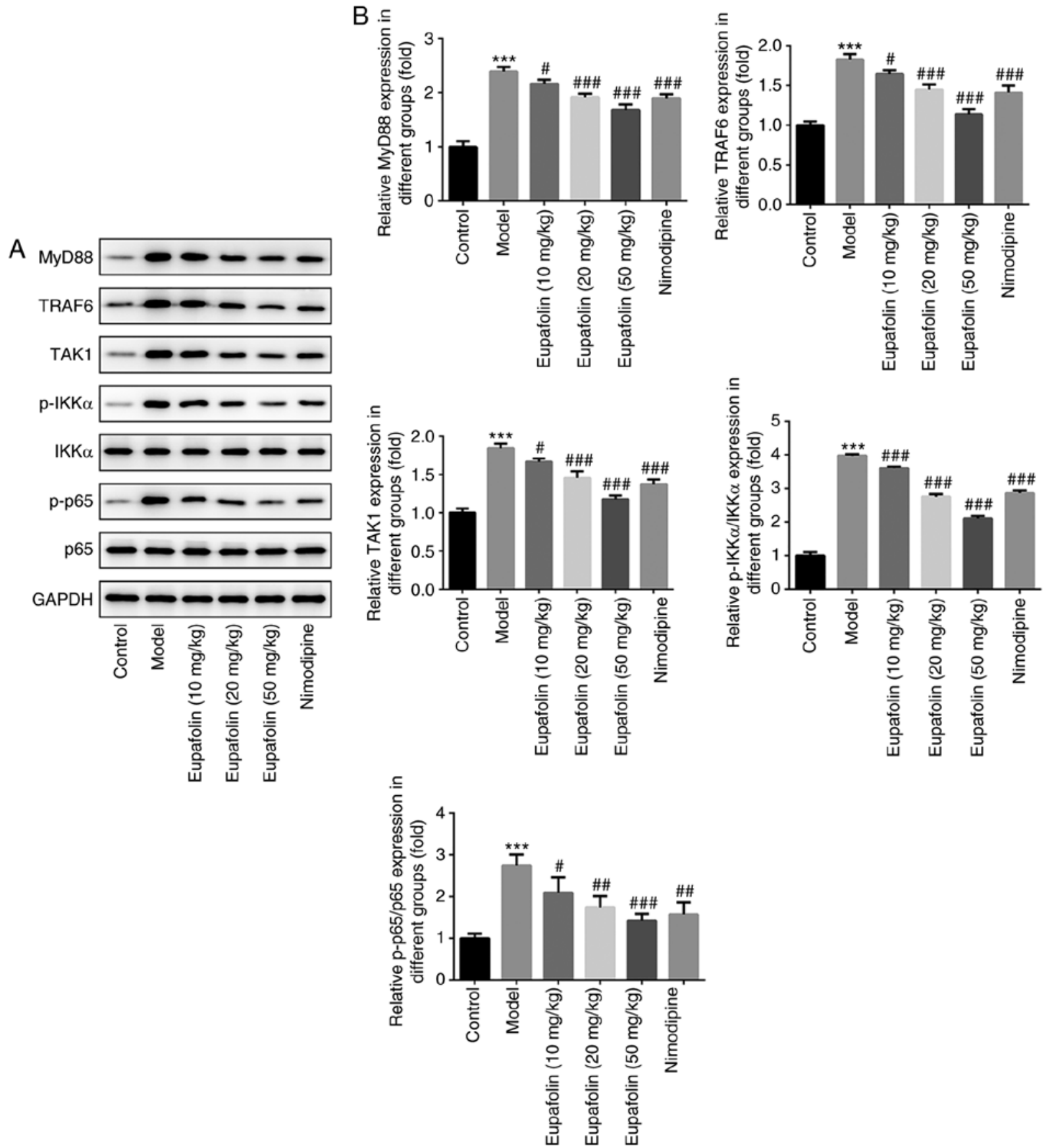

Figure 5. Expression of proteins involved in TLR4/NF- $\mathrm{kB}$ in brain tissues of rats from control, model, $10 \mathrm{mg} / \mathrm{kg}$ eupafolin, $20 \mathrm{mg} / \mathrm{kg}$ eupafolin, $50 \mathrm{mg} / \mathrm{kg}$ eupafolin and nimodipine groups. (A) Representative western blot bands for detecting MyD88, TRAF6, TAK1, p-IKK $\alpha /$ IKK $\alpha$ and p-p65/p65. GAPDH was used as loading control. (B) Densitometric quantification of protein expression. ${ }^{* * *} \mathrm{P}<0.001$ vs. control. ${ }^{\#} \mathrm{P}<0.05$, ${ }^{\# \#} \mathrm{P}<0.01$ and ${ }^{\# \# \#} \mathrm{P}<0.001$ vs. model. MyD88, myeloid differentiation factor 88; TRAF6, TNF receptor associated factor 6; TAK1, TGF-activated kinase 1; p, phosphorylated.

for the treatment of ischemic stroke. To date, in preclinical animal studies, $>700$ drugs have shown beneficial effects in cerebral ischemia, but the results are far from satisfactory (29). Eupafolin is a potent anti-inflammatory, antioxidant and antitumor agent, extracted from the traditional herb E. perfoliatum. Although eupafolin has been used as a traditional medicine for treating inflammatory-related diseases in China and India, the pharmacological research on it has only begun in the past ten years. Published reports have mainly focused on in vitro studies, and are lacking evidence from in vivo studies for clinical therapy (30). Jiang et al (14) demonstrated that eupafolin at $60 \mathrm{mg} / \mathrm{kg}$ significantly inhibited tumor growth and tumor angiogenesis in a hepatocellular carcinoma xenograft model. The present study investigated the protective effects of eupafolin on cerebral $\mathrm{I} / \mathrm{R}$ injury in rats and revealed that eupafolin at a dose of 10, 20 and $50 \mathrm{mg} / \mathrm{kg}$ all exhibited significant protective effects on cerebral I/R via inhibiting inflammation, oxidative stress and apoptosis. Concurrently, $20 \mathrm{mg} / \mathrm{kg}$ eupafolin showed nearly equivalent effects to nimodipine, which is used to improve blood circulation in the recovery period of acute cerebrovascular disease and has been proven to exert anti-inflammation and anti-apoptosis effects (31-33). These results provided in vivo data for the therapeutic effect of low-dose eupafolin in treating cerebral I/R injury. 
Table III. The neurological deficit score and brain edema evaluation in the absence or presence of TLR4 agonist-LPS.

\begin{tabular}{lccc}
\hline Group & Neurological deficit scores (range, 0-3) & Brain water content, \% & Brain index, \% \\
\hline Eupafolin (20 mg/kg) & $1.15 \pm 0.31$ & $83.04 \pm 0.53$ & $0.62 \pm 0.02$ \\
Eupafolin + LPS & $2.09 \pm 0.47^{\mathrm{b}}$ & $83.84 \pm 0.66^{\mathrm{a}}$ & $0.69 \pm 0.04^{\mathrm{b}}$ \\
\hline
\end{tabular}

The neurological deficit scores of rats among the eupafolin $20 \mathrm{mg} / \mathrm{kg}$ and eupafolin $20 \mathrm{mg} / \mathrm{kg}+\mathrm{LPS}$ groups were measured at $24 \mathrm{~h}$ after cerebral I/R $(\mathrm{n}=8)$. The brain samples of each group were harvested $24 \mathrm{~h}$ after cerebral $\mathrm{I} / \mathrm{R}$, and the brain water content and brain index were then measured $(\mathrm{n}=8)$. Data are presented as the mean \pm standard deviation. ${ }^{\mathrm{a}} \mathrm{P}<0.05$ and ${ }^{\mathrm{b}} \mathrm{P}<0.001 \mathrm{vs}$. eupafolin $20 \mathrm{mg} / \mathrm{kg}$ group. TLR, toll-like receptor; LPS, lipopolysaccharide; I/R, ischemia/reperfusion.
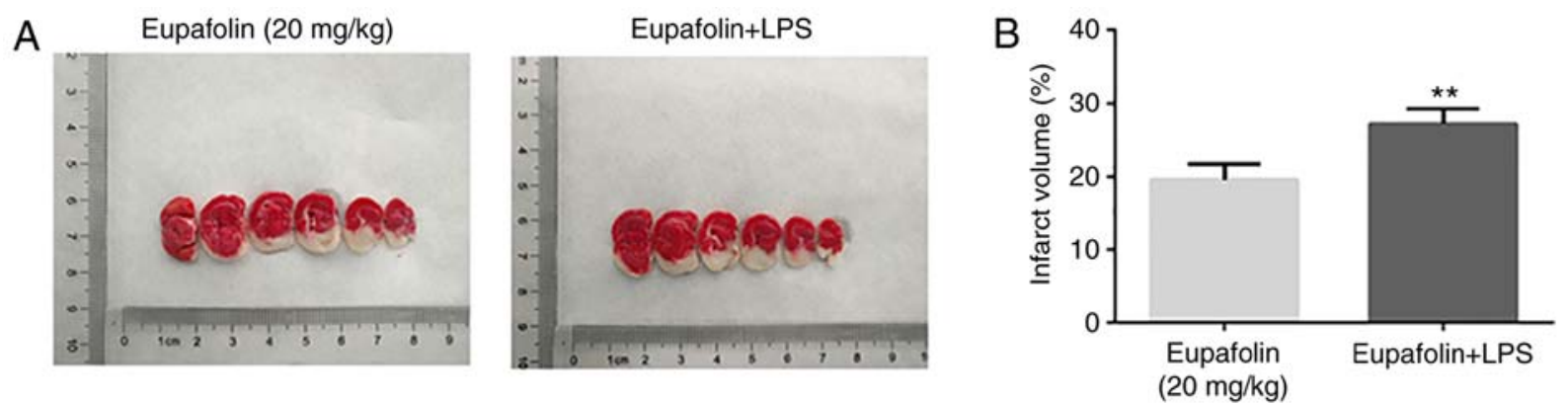

Figure 6. The presence of TLR4 agonist-LPS blunts the effect of eupafolin. (A) TCC staining images of brain tissues in rats at $24 \mathrm{~h}$ after cerebral I/R (B) Histogram of cerebral infarction volume in different groups. *** $\mathrm{P}<0.01 \mathrm{vs}$. eupafolin $20 \mathrm{mg} / \mathrm{kg}$. I/R, ischemia/reperfusion; TTC, 2,3,5-triphenyltetrazolium chloride; LPS, lipopolysaccharide.

Eupafolin may exert its actions via targeting multiple pathways, including NF- $\mathrm{KB}, \mathrm{PI} 3 \mathrm{~K} / \mathrm{AKT}$ and MAPK $(11,34,35)$. It is widely implicated that TLR4-MyD88 association may activate $\mathrm{NF}-\mathrm{\kappa B}$, which may activate neurons to secrete numerous pro-inflammatory mediators, including TNF- $\alpha$, IL-1 $\beta$ and IL-6, ultimately resulting in ischemic injury (36). The results of the present study demonstrated that cerebral I/R injury may significantly increase MyD88, TRAF6, TAK1, p-IKK $\alpha$ and p-p65 expression, and TNF- $\alpha$, IL-1 $\beta$ and IL- 6 levels. Following ligand binding, the toll/interleukin-1 receptor domain of TLR4 interacts with MyD88, thereby binding to TRAF6, resulting in the activation of TAK1. Activated TAK1 continues to signal via MAPK or NF- $\mathrm{B}$ (37). The present data indicated that eupafolin may prevent the activation of TLR4/NF- $\kappa B$ signaling. In addition, the neurological deficit score, brain edema and cerebral infarct size were decreased compared with the model group, indicating that the blockade of the TLR4/NK- $\mathrm{BB}$ signaling pathway in cerebral I/R may have a protective effect. Furthermore, the presence of LPS, which is publicly considered as the ligand of TLR4 and activates TLR4 signaling (38), markedly blunted the protective effect of $20 \mathrm{mg} / \mathrm{kg}$ eupafolin on neurological functions, brain edema and infarct volume of rats that underwent cerebral I/R injury, implicating that the actions of eupafolin may be at least partially dependent on the blocking of the TLR4/NF- $\mathrm{kB}$ signaling pathway.

However, the activation of TLR4 signaling is not only associated with the NF- $\mathrm{kB}$ pathway but is also associated with other processes, including the MAPK pathway. Therefore, the protective effects of eupafolin against cerebral I/R injury may not only be associated with the activation of the NF- $\mathrm{KB}$ pathway, but also with other mechanisms, which require further investigation. In addition, whether the inhibitory effect of eupafolin on inflammation, oxidative stress and apoptosis during cerebral I/R were dependent on blocking TLR4 signaling remain to be investigated. Meanwhile, the employment of TLR4-knockout or -knockdown mice is necessary to further confirm the results of the present study and to identify the underlying molecular mechanisms, and this will be performed in future experiments.

In conclusion, the results of the present study provided novel evidence that eupafolin exerted protective effects against cerebral I/R injury in rats exposed to MCAO followed by reperfusion. This may be associated with inhibiting the TLR4/NF- $\mathrm{BB}$ signaling pathway.

\section{Acknowledgements}

Not applicable.

\section{Funding}

No funding was received.

\section{Availability of data and materials}

All data generated or analyzed during this study are included in this published article.

\section{Authors' contributions}

JL and XC contributed toward study conception and design; $\mathrm{XC}, \mathrm{ZY}, \mathrm{XP}$ and LW contributed toward acquisition of data; HW and YO contributed toward analysis and interpretation of data; XC drafted the initial manuscript and JL revised it 
critically for important intellectual content. All authors read and approved the final manuscript.

\section{Ethics approval and consent to participate}

All experiments involving animals were approved by the Animal Studies Ethics Committees of the Shenzhen Hospital of Integrated Traditional Chinese and Western Medicine.

\section{Patient consent for publication}

Not applicable.

\section{Competing interests}

The authors declare that they have no competing interests.

\section{References}

1. Bustamante A and Montaner J: Author response: Usefulness of ADAMTS13 to predict response to recanalization therapies in acute ischemic stroke. Neurology 91: 899, 2018.

2. Kahl A, Blanco I, Jackman K, Baskar J, Mohan HM, Rodney-Sandy R, Zhang S, Iadecola C and Hochrainer K: Cerebral ischemia induces the aggregation of proteins linked to neurodegenerative diseases. Sci Rep 8: 2701, 2018.

3. Huang J, Wang T, Yu D, Fang X, Fan H, Liu Q, Yi G, Yi X and Liu Q: 1-homocarnosine attenuates inflammation in cerebral ischemia-reperfusion injury through inhibition of nod-like receptor protein 3 inflammasome. Int $\mathbf{J}$ Biol Macromol 118 357-364, 2018

4. Zhang H, Park JH, Maharjan S, Park JA, Choi KS, Park H, Jeong Y, Ahn JH, Kim IH, Lee JC, et al: Sac-1004, a vascular leakage blocker, reduces cerebral ischemia-reperfusion injury by suppressing blood-brain barrier disruption and inflammation. J Neuroinflammation 14: 122, 2017.

5. Ya BL, Liu Q, Li HF, Cheng HJ, Yu T, Chen L, Wang Y, Yuan LL, Li WJ, Liu WY and Bai B: Uric acid protects against focal cerebral ischemia/reperfusion-induced oxidative stress via activating $\mathrm{Nrf} 2$ and regulating neurotrophic factor expression. Oxid Med Cell Longev 2018: 6069150, 2018.

6. Li X, Cheng S, Hu H, Zhang X, Xu J, Wang R and Zhang P: Progranulin protects against cerebral ischemia-reperfusion (I/R) injury by inhibiting necroptosis and oxidative stress. Biochem Biophys Res Commun 521: 569-576, 2020.

7. Cui Y, Wang JQ, Shi XH, Wang YY, Liu HY, Li Z, Dong Y, Mang J and Xu ZX: Nodal mitigates cerebral ischemia-reperfusion injury via inhibiting oxidative stress and inflammation. Eur Rev Med Pharmacol Sci 23: 5923-5933, 2019.

8. Lee CW, Lin ZC, Hsu LF, Fang JY, Chiang YC, Tsai MH, Lee MH, Li SY, Hu SC, Lee IT and Yen FL: Eupafolin ameliorates COX-2 expression and PGE2 production in particulate pollutants-exposed human keratinocytes through ROS/MAPKs pathways. J Ethnopharmacol 189: 300-309, 2016.

9. Maas M, Deters AM and Hensel A: Anti-inflammatory activity of Eupatorium perfoliatum L. Extracts, eupafolin, and dimeric guaianolide via iNOS inhibitory activity and modulation of inflammation-related cytokines and chemokines. J Ethnopharmacol 137: 371-381, 2011.

10. Zhang H, Chen MK, Li K, Hu C, Lu MH and Situ J: Eupafolin nanoparticle improves acute renal injury induced by LPS through inhibiting ROS and inflammation. Biomed Pharmacother 85 704-711, 2017.

11. Gao Y, Zhang Y and Fan Y: Eupafolin ameliorates lipopolysaccharide-induced cardiomyocyte autophagy via PI3K/AKT/mTOR signaling pathway. Iran J Basic Med Sci 22: 1340-1346, 2019

12. Sung HC, Liang CJ, Lee CW, Yen FL, Hsiao CY, Wang SH, Jiang-Shieh YF, Tsai JS and Chen YL: The protective effect of eupafolin against TNF- $\alpha$-induced lung inflammation via the reduction of intercellular cell adhesion molecule-1 expression. J Ethnopharmacol 170: 136-147, 2015.
13. Fan X, Tao J, Cai Z, Fredimoses M, Wu J, Jiang Z, Zhang K and Li S: Eupafolin suppresses esophagus cancer growth by targeting T-LAK cell-originated protein kinase. Front Pharmacol 10: 1248,2019

14. Jiang H, Wu D, Xu D, Yu H, Zhao Z, Ma D and Jin J: Eupafolin exhibits potent anti-angiogenic and antitumor activity in hepatocellular carcinoma. Int J Biol Sci 13: 701-711, 2017.

15. Han MA, Min KJ, Woo SM, Seo BR and Kwon TK Eupafolin enhances TRAIL-mediated apoptosis through cathepsin S-induced down-regulation of $\mathrm{Mcl}-1$ expression and AMPK-mediated Bim up-regulation in renal carcinoma Caki cells. Oncotarget 7: 65707-65720, 2016.

16. Liu K, Park C, Chen H, Hwang J, Thimmegowda NR, Bae EY, Lee KW, Kim HG, Liu H, Soung NK, et al: Eupafolin suppresses prostate cancer by targeting phosphatidylinositol 3-kinasemediated Akt signaling. Mol Carcinog 54: 751-760, 2015

17. Lester SN and Li K: Toll-like receptors in antiviral innate immunity. J Mol Biol 426: 1246-1264, 2014.

18. Hua F, Ma J, Ha T, Xia Y, Kelley J, Williams DL, Kao RL, Browder IW, Schweitzer JB, Kalbfleisch JH and Li C: Activation of toll-like receptor 4 signaling contributes to hippocampal neuronal death following global cerebral ischemia/reperfusion. J Neuroimmunol 190: 101-111, 2007.

19. van Delft MA, Huitema LF and Tas SW: The contribution of $\mathrm{NF}-\kappa \mathrm{B}$ signalling to immune regulation and tolerance. Eur J Clin Invest 45: 529-539, 2015.

20. Liang W, Lin C, Yuan L, Chen L, Guo P, Li P, Wang W and Zhang X: Preactivation of Notch1 in remote ischemic preconditioning reduces cerebral ischemia-reperfusion injury through crosstalk with the NF-kB pathway. J Neuroinflammation 16: 181, 2019.

21. Auvin S, Shin D, Mazarati A and Sankar R: Inflammation induced by LPS enhances epileptogenesis in immature rat and may be partially reversed by IL1RA. Epilepsia 51 (Suppl 3): S34-S38, 2010.

22. Zhao R, Jiang J, Li H, Chen M, Liu R, Sun S, Ma D, Liang X and Wang S: Phosphatidylserine-microbubble targeting-activated microglia/macrophage in inflammation combined with ultrasound for breaking through the blood-brain barrier. J Neuroinflammation 15: 334, 2018.

23. Sommer CJ: Ischemic stroke: Experimental models and reality. Acta Neuropathol 133: 245-261, 2017.

24. Li P, Zhang Y and Liu H: The role of $\mathrm{Wnt} / \beta$-catenin pathway in the protection process by dexmedetomidine against cerebral ischemia/reperfusion injury in rats. Life Sci 236: 116921, 2019.

25. Bederson JB, Pitts LH, Tsuji M, Nishimura MC, Davis RL and Bartkowski H: Rat middle cerebral artery occlusion: Evaluation of the model and development of a neurologic examination. Stroke 17: 472-476, 1986

26. Yang Z, Weian C, Susu H and Hanmin W: Protective effects of mangiferin on cerebral ischemia-reperfusion injury and its mechanisms. Eur J Pharmacol 771: 145-151, 2016.

27. Gong L, Tang Y, An R, Lin M, Chen L and Du J: RTN1-C mediates cerebral ischemia/reperfusion injury via ER stress and mitochondria-associated apoptosis pathways. Cell Death Dis 8: e3080, 2017.

28. Jing H, Liu L, Jia Y, Yao H and Ma F: Overexpression of the long non-coding RNA Oprm1 alleviates apoptosis from cerebral ischemia-reperfusion injury through the Oprm1/miR-155/GATA3 axis. Artif Cells Nanomed Biotechnol 47: 2431-2439, 2019.

29. Yamashita T and Abe K: Recent progress in therapeutic strategies for ischemic stroke. Cell Transplant 25: 893-898, 2016.

30. Hensel A, Maas M, Sendker J, Lechtenberg M, Petereit F, Deters A, Schmidt T and Stark T: Eupatorium perfoliatum L.: Phytochemistry, traditional use and current applications. J Ethnopharmacol 138: 641-651,2011.

31. Sanz JM, Chiozzi P, Colaianna M, Zotti M, Ferrari D, Trabace L, Zuliani G, Di Virgilio F: Nimodipine inhibits IL-1 $\beta$ release stimulated by amyloid $\beta$ from microglia. Br J Pharmacol 167 1702-1711, 2012

32. Wang GH, Liu Y, Wu XB, Lu Y, Liu J, Qin YR, Li T and Duan HF: Neuroprotective effects of human umbilical cord-derived mesenchymal stromal cells combined with nimodipine against radiation-induced brain injury through inhibition of apoptosis Cytotherapy 18: 53-64, 2016

33. Yan B, Sun Y, Zeng J, Chen Y, Li C, Song P, Zhang L, Yang X, $\mathrm{Wu} Y$ and $\mathrm{Ma}$ P: Combined use of vitamin $\mathrm{E}$ and nimodipine ameliorates dibutyl phthalate-induced memory deficit and apoptosis in mice by inhibiting the ERK 1/2 pathway. Toxicol Appl Pharmacol 368: 1-17, 2019. 
34. Ko HH, Chiang YC, Tsai MH, Liang CJ, Hsu LF, Li SY, Wang MC, Yen FL and Lee CW: Eupafolin, a skin whitening flavonoid isolated from Phyla nodiflora, downregulated melanogenesis: Role of MAPK and Akt pathways. J Ethnopharmacol 151: 386-393, 2014.

35. Chen CC, Lin MW, Liang CJ and Wang SH: The anti-inflammatory effects and mechanisms of eupafolin in lipopolysaccharide-induced inflammatory responses in RAW264.7 macrophages. PLoS One 11: e0158662, 2016.

36. Luo SY, Li R, Le ZY, Li QL and Chen ZW: Anfibatide protects against rat cerebral ischemia/reperfusion injury via TLR4/JNK/caspase-3 pathway. Eur J Pharmacol 807: 127-137, 2017.
37. de Groen RAL, Schrader AMR, Kersten MJ, Pals ST and Vermaat JSP: MYD88 in the driver's seat of B-cell lymphomagenesis: From molecular mechanisms to clinical implications. Haematologica 104: 2337-2348, 2019.

38. Araya EI, Barroso AR, Turnes JM, Radulski DR, Jaganaught JA, Zampronio AR and Chichorro JG: Toll-like receptor 4 (TLR4) signaling in the trigeminal ganglion mediates facial mechanical and thermal hyperalgesia in rats. Physiol Behav 226: 113127,2020.

(i) (3) This work is licensed under a Creative Commons Attribution-NonCommercial-NoDerivatives 4.0 International (CC BY-NC-ND 4.0) License. 